\title{
Phenotype and Genotype Characterization and Twin Association in Patients with Anderson-Fabry Cardiomyopathy
}

\author{
Miquel Gomez Lluis Molina Mercedes Cladellas Soledad Ascoeta \\ Cristina Soler Mireia Ble Aleyska Ramirez Jordi Bruguera \\ Cardiology Department, Hospital del Mar-Parc de Salut Mar, Barcelona, Spain
}

\section{Key Words}

Anderson-Fabry disease $\cdot$ Twins $\cdot$ GLA gene $\cdot$

Cardiomyopathy

\begin{abstract}
Anderson-Fabry disease (FD), an X-linked recessive lysosomal storage disorder caused by a deficiency of $\alpha$-galactosidase A ( $\alpha$-Gal A) activity, is associated with cardiac manifestations including arrhythmias, valvular abnormalities, and cardiomyopathy. Early initiation of enzyme replacement therapy (ERT) may have the potential to delay the underlying clinical outcomes in patients with FD. Clinical electrocardiogram (ECG) and echocardiography were used to characterize the cardiomyopathy. Diagnosis of FD was performed by measuring the $\alpha$-Gal A activity in plasma and mutation analysis by direct sequencing using capillary electrophoresis. We identified four adult hemizygous male patients with cardiomyopathy and other symptoms related to FD; two of them were monozygotic twins. In all cases, ECG and echocardiography showed severe left ventricular (LV) hypertrophy. Some years later, all patients showed typical symptoms of FD, including angiokeratomas and neurological, renal, gastrointestinal, and ocular involvement. A deficiency of $\alpha$-Gal A activity and point mutations in exon 5 of the GLA gene were detected in
\end{abstract}

all patients. ERT (agalsidase-alfa) was administered every other week as a $0.2 \mathrm{mg} / \mathrm{kg}$ intravenous infusion over $40 \mathrm{~min}$. In conclusion, these findings highlight the importance of screening middle-aged patients with LV hypertrophy for the early detection of FD, particularly in direct-line relatives such as twins.

Copyright $\odot 2012$ S. Karger AG, Basel

\section{Introduction}

Anderson-Fabry disease (FD) is a rare inherited Xlinked metabolic lysosomal storage disorder caused by an $\alpha$-galactosidase A ( $\alpha$-Gal A) deficiency, characterized by progressive lysosomal accumulation of lipids (glycosphingolipids, predominantly globotriaosylceramide) in a variety of cell types (including renal and myocardial cells, heart valve fibrocytes, neurons, and endothelial smooth cells of blood vessels) [1-4].

The estimated incidence of FD is 1 in 117,000 in the general population and 1 in 40,000 in males [1], primarily affecting hemizygous males and heterozygous females, the lifespan of whom is typically reduced by approximately $15-20$ years [1].

\section{KARGER}

Fax +4161306 1234

E-Mail karger@karger.ch

www.karger.com
(C) 2012 S. Karger AG, Basel

$0008-6312 / 12 / 1212-0071 \$ 38.00 / 0$

Accessible online at:

www.karger.com/crd 
FD is associated with a broad range of clinical symptoms, including: pain and paresthesias in the extremities; sensorineural hearing loss; angiokeratoma; corneal and lenticular opacities; hypohidrosis; gastrointestinal, cardiac, and renal dysfunction, and transient ischemic attack/stroke [1]. The typical features of Fabry's cardiomyopathy have been investigated in recent years; the early stage is characterized by left ventricular (LV) hypertrophy and reduced regional myocardial function [4].

Biochemical diagnosis of FD is made by measuring the $\alpha-G a l$ A activity in peripheral leucocytes or plasma and genetic confirmation is achieved by $\alpha-\mathrm{Gal}$ A gene (GLA) sequencing [5]. Mutation analysis of the GLA gene is a valuable tool for the accurate diagnosis of affected families $[6,7]$. Disease onset and progression can be monitored by clinical, radiological, and laboratory assessments [8].

Once a diagnosis is made, FD can be effectively and safely treated with enzyme replacement therapy (ERT) [1], available since 2001, allowing a variety of clinical benefits, including improved renal pathology/function and cardiac function [3]. However, this progressive multipleorgan disease results in frequent misdiagnosis and diagnostic delay, which may impact patient outcomes [1].

Since FD is a relatively prevalent cause of LV hypertrophy [6] (particularly among middle-aged patients) [9], it seems reasonable to screen these patients for $\alpha$-Gal A deficiency and gene mutation [9].

In this context, we report four hemizygous male patients diagnosed with FD after suffering from LV hypertrophy, further characterized as infiltrative cardiomyopathy due to deposition of glycosphingolipids.

\section{Methods}

Patient characteristics, including clinical symptoms, age at onset of symptoms, and previous diagnoses were collected. Experienced personnel performed electrocardiograms (ECG) and complete echocardiography including tissue Doppler imaging (TDI) to characterize Fabry's cardiomyopathy. The LV wall thickness (interventricular septum/posterior wall), LV mass index (LVMI; normal values $<125 \mathrm{~g} / \mathrm{m}^{2}$ ), and ejection fraction (using the modified Simpson method) were calculated. The LVMI was calculated using Devereux's formula [10] considering the diastolic measurements of LV internal diameter (LVID), interventricular septal thickness (IVST), and posterior wall thickness (PWT) as follows: LVMI $\left(\mathrm{g} / \mathrm{m}^{2}\right)=(1.04 \text { [(IVST + LVID + PWT })^{3}-$ $\left.\mathrm{LVID}^{3}\right]-14 \mathrm{~g}$ )/body surface area. Renal function was assessed by serum creatinine testing and creatinine clearance (the CockcroftGault equation was used to estimate the glomerular filtration rate).
FD diagnosis was performed based on the measurement of $\alpha$ Gal A activity in peripheral leucocytes and plasma (reference levels $2.1-13.6 \mu \mathrm{mol} / \mathrm{h} / \mathrm{l}$ ) and it was genetically confirmed by detection of point mutations in exon 5 of the GLA gene by means of direct sequencing using capillary electrophoresis.

\section{Case Reports}

We report four adult hemizygous male childless patients who were diagnosed with FD after suffering from LV hypertrophy, further characterized as infiltrative cardiomyopathy due to deposition of glycosphingolipids. In all four patients, systolic function was within normal ranges and diastolic function was impaired (abnormality of ventricular relaxation objectified by TDI in the lateral mitral annulus) and the ECG showed typical signs of LV hypertrophy with deep negative $\mathrm{T}$ waves in lateral derivations (DI, aVL, V4, V5, and V6) (table 1).

Some years after (between 1 and 5 years, approximately) the cardiomyopathy diagnosis, all patients showed a phenotype indicative of FD (neurological, renal, gastrointestinal, and ocular disorders and angiokeratomas) and diagnosis was performed based on the measurement of $\alpha$-Gal A activity and genotyping (table 1). At this stage, ERT was established in the outpatient facilities, consisting of intravenous infusions of agalsidase-alfa ( $\mathrm{Re}$ plagal; Shire Human Genetic Therapies AB, Sweden) at $0.2 \mathrm{mg} / \mathrm{kg}$ every other week (during 3 years in patient 1, 6 years in patient 2, and 6 months in patient 3 ).

\section{Patient 1}

At the age of 59 years, he was diagnosed with severe LV hypertrophy, based on echocardiographic exploration (table 1; fig. 1) and an ECG (atrial fibrillation and blockage of the left branch). He had a history of mild hypertension and his family history was unremarkable.

Seven years later, characteristic symptoms of FD were detected, including angiokeratomas, neurological affectation (ischemic stroke with complete recovery, hypo/anhydrosis), gastrointestinal disorders, signs of corneal depositions (cornea verticillata), muscular pain, and renal involvement (chronic renal failure requiring dialysis and, finally, renal transplantation) (table 1). FD diagnosis was performed based on the deficiency of $\alpha$-Gal A activity $(0.92$ $\mu \mathrm{mol} / \mathrm{h} / \mathrm{l})$ and detection of a nonsense mutation in exon 5 of the GLA gene: R227X (c.C679>T) (table 1). After a 4-year period of ERT, the patient was stable (echocardiographically objectified by a $5-\mathrm{mm}$ regression in $\mathrm{LV}$ wall thickness) and atrial fibrillation persisted.

\section{Patient 2}

A similar case was described when, at the age of 40 years, a man was diagnosed with mild LV hypertrophy based on echocardiographic exploration (fig. 1; table 1) and an ECG (sinusal dysfunction) (table 1). No other remarkable personal or familial history of diseases was registered.

Seven years later, at the age of 47 years, this patient presented symptoms indicative of FD, including neurological signs (neuropathic pain, hand paresthesias, and hypo/anhydrosis), angiokeratomas, gastrointestinal disorders, muscular pain, and renal involvement (table 1$)$. Deficiency of $\alpha$-Gal A activity $(0.80 \mu \mathrm{mol} /$ 
Table 1. Characteristics of patients

\begin{tabular}{|c|c|c|c|c|}
\hline \multirow[t]{2}{*}{ Variable } & \multicolumn{4}{|c|}{ Patient No. } \\
\hline & 1 & 2 & 3 & 4 \\
\hline \multicolumn{5}{|l|}{ Demographic characteristics } \\
\hline Sex & M & M & M & M \\
\hline Age at cardiomyopathy diagnosis, years & 59 & 40 & 47 & 47 \\
\hline Age at FD diagnosis, years & 66 & 47 & 49 & 49 \\
\hline Creatinine levels, mg/dl & 6.0 & 2.0 & 6.7 & 4.0 \\
\hline Creatinine clearance, $\mathrm{ml} / \mathrm{min} / 1.73 \mathrm{~m}^{2}$ & 10 & 43 & 7 & 16 \\
\hline \multicolumn{5}{|l|}{ Electrocardiographic characteristics } \\
\hline \multicolumn{5}{|l|}{ Signs of LV hypertrophy with deep negative T waves in lateral derivations } \\
\hline$(\mathrm{DI}, \mathrm{aVL}, \mathrm{V} 4, \mathrm{~V} 4, \mathrm{~V} 6)$ & + & + & + & + \\
\hline Atrial fibrillation and blockage of the left branch & + & 0 & 0 & 0 \\
\hline Sinusal dysfunction (asymptomatic sinus bradycardia at 45 beats/min) & 0 & + & 0 & 0 \\
\hline Ventricular fibrillation & 0 & 0 & 0 & + \\
\hline \multicolumn{5}{|l|}{ Echocardiographic characteristics } \\
\hline LV wall thickness (interventricular septum/posterior wall), mm & $21 / 21$ & $14 / 14$ & $24 / 21$ & $24 / 24$ \\
\hline VMS $\left(\mathrm{g} / \mathrm{m}^{2}\right)$ & 317 & 143 & 303 & 352 \\
\hline Ejection fraction, \% & 60 & 65 & 55 & 60 \\
\hline Abnormality of ventricular relaxation in the lateral mitral annulus (TDI) & + & + & + & + \\
\hline \multicolumn{5}{|l|}{ Renal involvement } \\
\hline Creatinine levels, mg/dl & 6.0 & 2.0 & 6.7 & 4.0 \\
\hline Creatinine clearance, $\mathrm{ml} / \mathrm{min} / 1.73 \mathrm{~m}^{2}$ & 10 & 43 & 7 & 16 \\
\hline Dialysis & + & 0 & 0 & 0 \\
\hline Renal transplantation & + & 0 & 0 & 0 \\
\hline \multicolumn{5}{|l|}{ Neurological symptoms } \\
\hline Ischemic stroke & + & 0 & 0 & 0 \\
\hline Neuropathic pain and paresthesias & 0 & + & 0 & 0 \\
\hline Postanoxic encephalopathy & 0 & 0 & 0 & + \\
\hline \multicolumn{5}{|l|}{ Angiokeratomas } \\
\hline Periumbilical area & + & + & + & + \\
\hline Buttocks & + & + & 0 & 0 \\
\hline Legs & 0 & + & + & + \\
\hline Back & + & 0 & yes & yes \\
\hline \multicolumn{5}{|l|}{ Gastrointestinal disorders } \\
\hline Diarrhea & + & 0 & 0 & 0 \\
\hline Abdominal pain & + & + & + & + \\
\hline Precocious satiety & + & + & + & + \\
\hline Nausea & + & + & + & + \\
\hline Vomiting & + & + & + & + \\
\hline \multicolumn{5}{|l|}{ Ocular symptoms } \\
\hline Cornea verticillata & + & 0 & 0 & 0 \\
\hline Muscular pain & + & + & + & no \\
\hline$\alpha$-Gal A activity, $\mu \mathrm{mol} / \mathrm{h} / \mathrm{l}$ & 0.92 & 0.80 & 1.05 & 0.68 \\
\hline \multicolumn{5}{|l|}{ Mutation in exon 5 of the GLA gene } \\
\hline Nonsense R227X (c.C679>T) & + & 0 & + & + \\
\hline Missense W81S (c.242G>C) & 0 & + & 0 & 0 \\
\hline
\end{tabular}

$\mathrm{h} / \mathrm{l})$ and detection of a missense mutation in exon 5 of the GLA gene (W81S>C, c.242G>C) were found (table 1; fig. 2).

Since ERT was started at an early stage of the cardiomyopathy, after a 7-year treatment period, echocardiographic exploration showed stabilization of LV wall thickness and absence of progression to hypertrophy.

FD Phenotype and Genotype in Twins
Patient 3

Because of a familiar study of his monozygotic twin son (case 4), at the age of 47 years, patient 3 was diagnosed with severe LV hypertrophy (table 1; fig. 1).

Two years later, characteristic symptoms of FD, including angiokeratomas, gastrointestinal disorders, neurological symptoms 


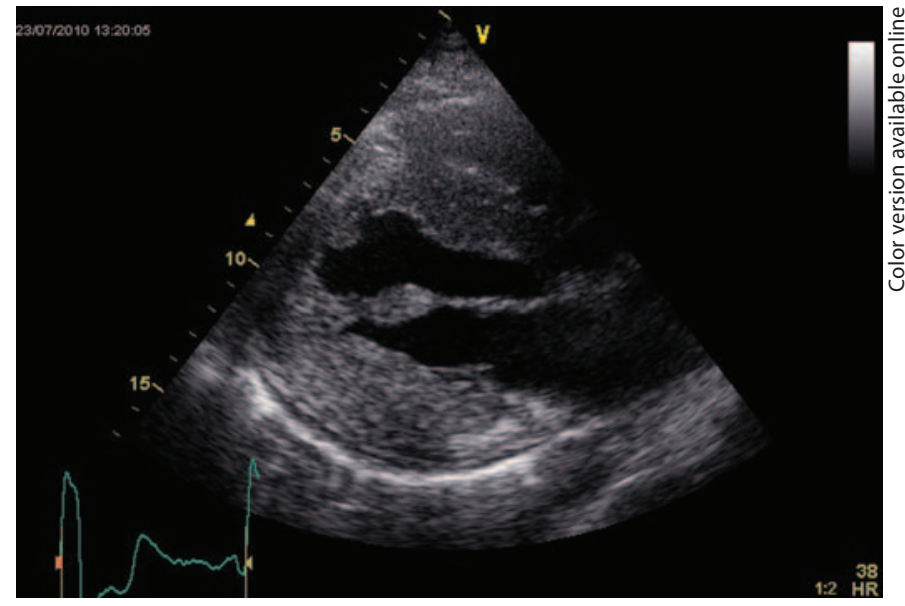

Fig. 1. Instrumental features of case 1. Typical echocardiographic pattern of LV hypertrophy in FD (severe concentric LV hypertrophy).

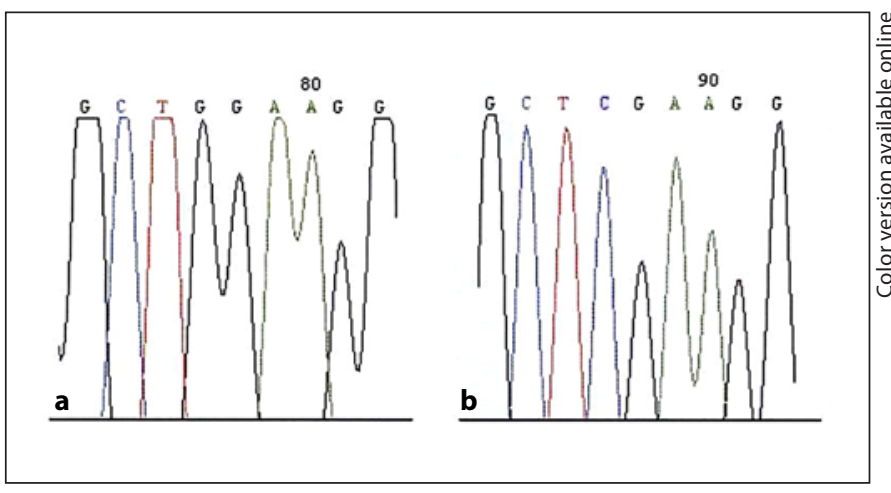

Fig. 2. DNA sequencing of exon 5 of the GLA gene of patient 2 . Mutation W81S in which a cytosine substitutes a guanine. The patient is heterozygous for the mutation. a Normal sequence. b Patient sequence.

(hypo/anhydrosis), muscular pain, and renal involvement (table 1) were observed. FD disease was diagnosed based on the deficiency of $\alpha$-Gal A activity $(1.05 \mu \mathrm{mol} / \mathrm{h} / \mathrm{l})$ and detection of a nonsense mutation in exon 5 of the GLA gene: R227X (c.C679>T) (table 1). After a 5-month period of ERT, no changes were observed in the cardiomyopathy.

\section{Patient 4}

At the age of 47 years, patient 4 experienced sudden cardiac death and recovered by primary ventricular fibrillation requiring electrical cardioversion; as an adverse outcome, the patient suffered from postanoxic encephalopathy (with severe functional and cognitive impairment). Echocardiographic and ECG findings allowed the diagnosis of severe LV hypertrophy (with ventricular fibrillation) (table 1; fig. 1). FD was searched for and diagnosed based on characteristic symptoms [angiokeratomas, gastrointestinal disorders, neurological symptoms (hypo/anhydrosis), and renal involvement], $\alpha$-Gal A activity $(0.68 \mu \mathrm{mol} / \mathrm{h} / \mathrm{l})$, and genotype (the same mutation as in case 3) (table 1). Due to the presence of postanoxic encephalopathy, ERT was not initiated.

\section{Discussion}

Although FD (the classical phenotype) is often associated with renal dysfunction and death due to renal failure in middle-aged patients, cardiac manifestations are also common and probably underrecognized [9]. Furthermore, a variant of FD has been identified that only affects cardiac tissue, which presents as an unexplained LV hypertrophy in middle-aged patients [9].

In this regard, the observed cardiac involvement of our patients perfectly correlates with the reported cardiac symptoms in FD patients, characterized by arrhythmias and LV hypertrophy [4, 9].

Moreover, we also observed early signs and symptoms characteristic of enzymatic defect [9], including angiokeratomas, neurological symptoms, and corneal lesions. Our patients also presented renal impairment, another serious manifestation of FD, leading to the gradual deterioration of renal function and end-stage renal disease. These findings also agree with recent data from the Fabry Outcome Survey (FOS), according to which the predominant clinical involvement in Spanish male patients was renal (69\%), cardiac (66\%), and neurological (60\%) [11].

As recommended [12], our cases were diagnosed demonstrating the deficiency of $\alpha$-Gal A and genetic mutations of the GLA gene, which is located on the X-chromosome and contains 7 introns and 7 exons. To date, more than 600 pathogenetic mutations (missense, nonsense, and splice site point mutations and 'short length' rearrangements such as deletions and duplications) and up to 14 DNA polymorphisms have been reported [2].

In our report, two point mutations in exon 5 were identified: R227X in patients 1, 3, and 4 and W81S in patient 2. The missense mutation W81S (c.242G $>$ C) was first identified in a study performed in Spanish patients with FD [13], in which mutation R227X was also described. The R227X mutation involves CpG dinucleotides, which are hot spots for mutation, and is one of the most common mutations causing the classic phenotype of the disease [12].

Further studies are required to correlate these mutations with FD phenotypes and severity. However, the analysis of genotype-phenotype correlations in Fabry disease is complicated by a number of factors, such as the high 
proportion of private mutations, the large phenotypic heterogeneity associated with the same mutation, and the presence of FD-related complications that are observed with a high prevalence in the general population [8].

A notable finding of this study was the identification of FD in male twins who had the same point mutation (R227X). To date, a few cases of twins with FD have been reported, more in females [14] than in males [15]. Cases of female monozygotic twins discordant for FD are described, thus having important consequences for genetic counseling [8]. To date, male twins have only been identified in a mutation analysis performed in newborns [14].

Therefore, we present for the first time the case of adult male monozygotic twins who had both the same mutation (R227X) and a similar phenotype.

This case highlights the importance of early screening of direct relatives upon FD diagnosis. Moreover, given that epidemiological data indicates a prevalence of $1-12 \%$ of FD-related cardiomyopathy among middle-aged patients with cardiac hypertrophy, it is reasonable to screen these patients for $\alpha-G a l$ A deficiency and mutation analysis [9].

Moreover, in men diagnosed with LV hypertrophy, cardiac variants of FD are not exceptional and might ben- efit from ERT. As the outcome of ERT is less efficient in advanced cases of FD, before extensive myocardial fibrosis is present, screening these patients appears to be clinically relevant.

In conclusion, we have identified four adult hemizygous male patients, including two monozygotic twins, with cardiomyopathy and other symptoms related to FD, highlighting the importance of screening middle-aged patients with cardiac hypertrophy for the early detection of FD, particularly, in direct relatives such as twins.

\section{Acknowledgements}

We would like to express our gratitude to Nuria Piqué, $\mathrm{PhD}$, for her help in the preparation of the manuscript. The authors received editorial help from Adelphi and were supported by Shire Pharmaceuticals Spain with an unrestricted educational grant. Shire HGT funded Adelphi for the medical writing support.

\section{Conflict of Interest}

This paper was funded by Shire Pharmaceuticals Ibérica.

\section{References}

1 Lidove O, Kaminsky P, Hachulla E, et al: Fabry disease 'The New Great Imposter': results of the French Observatoire in Internal Medicine Departments (FIMeD). Clin Genet 2011, DOI: $10.1111 /$ j.1399-0004.2011.01718.x.

2 Ferri L, Guido C, la Marca G, et al: Fabry disease: polymorphic haplotypes and a novel missense mutation in the GLA gene. Clin Genet 2011, DOI: 10.1111/j.1399-0004.2011. 01689.x.

$>_{3}$ Weidemann F, Niemann M, Breunig F, Herrmann S, Beer M, Störk S, Voelker W, Ertl G, Wanner C, Strotmann J: Long-term effects of enzyme replacement therapy on Fabry cardiomyopathy: evidence for a better outcome with early treatment. Circulation 2009;119:524-529.

-4 Wu JC, Ho CY, Skali H, Abichandani R, Wilcox WR, Banikazemi M, Packman S, Sims K, Solomon SD: Cardiovascular manifestations of Fabry disease: relationships between left ventricular hypertrophy, disease severity, and alpha-galactosidase A activity. Eur Heart J 2010;31:1088-1097.

5 Massaccesi L, Burlina A, Baquero CJ, et al: Whole-blood alpha-D-galactosidase A activity for the identification of Fabry's patients. Clin Biochem 2011;44:916-921.
-6 Yoshimitsu M, Higuchi K, Miyata M, et al: Identification of novel mutations in the $\alpha$ galactosidase A gene in patients with Fabry disease: pitfalls of mutation analyses in patients with low $\alpha$-galactosidase A activity. J Cardiol 2011;57:345-353.

7 Aerts JM, Kallemeijn WW, Wegdam W, et al: Biomarkers in the diagnosis of lysosomal storage disorders: proteins, lipids, and inhibodies. J Inherit Metab Dis 2011;34:605619.

8 Ries M, Gal A: Genotype-phenotype correlation in Fabry disease; in Mehta A, Beck M, Sunder-Plassmann G (eds): Fabry Disease: Perspectives from 5 Years of FOS. Oxford, Oxford PharmaGenesis, 2006, chapter 34.

-9 Morrissey RP, Philip KJ, Schwarz ER: Cardiac abnormalities in Anderson-Fabry disease and Fabry's cardiomyopathy. Cardiovasc J Afr 2011;22:38-44.

10 Devereux RB, Reichek N: Echocardiographic determination of left ventricular mass in man: anatomic validation of the method. Circulation 1997;55:613-619.
-11 Barba-Romero MA, Rivera-Gallego A, Pintos-Morell G, Spanish FOS-Study Group: Fabry disease in Spain: description of Spanish patients and a comparison with other European countries using data from the Fabry Outcome Survey (FOS). Int J Clin Pract 2011; 65:903-910.

12 Winchester B, Young E: Biochemical and genetic diagnosis of Fabry disease; in Mehta A, Beck M, Sunder-Plassmann G (eds): Fabry Disease: Perspectives from 5 Years of FOS Oxford, Oxford PharmaGenesis, 2006, chapter 18.

13 Rodríguez-Marí A, Coll MJ, Chabás A: Molecular analysis in Fabry disease in Spain: fifteen novel GLA mutations and identification of a homozygous female. Hum Mutat 2003; 22:258.

14 Marguery MC, Giordano F, Parant M, et al: Fabry's disease: heterozygous form of different expression in two monozygous twin sisters. Dermatology 1993;187:9-15.

15 Hwu WL, Chien YH, Lee NC, et al: Newborn screening for Fabry disease in Taiwan reveals a high incidence of the later-onset GLA mutation c. 936 + 919G $>$ A (IVS4 + 919G $>$ A) Hum Mutat 2009;30:1397-1405. 